\title{
émulations
}

\section{Rémi Dormois - Les politiques urbaines. Histoire et enjeux contemporains}

\section{Antoine Lévêque}

Émulations - Revue de sciences sociales

2017, «Comptes rendus critiques, En ligne»

\section{Article disponible à l'adresse suivante}

https://ojs.uclouvain.be/index.php/emulations/article/view/7273

\section{Pour citer cet article}

Antoine Lévêque, « Rémi Dormois — Les politiques urbaines. Histoire et enjeux contemporains », Émulations, en ligne. Mise en ligne le 9 février 2017.

DOI : 10.14428/emulations.cr.028

Distribution électronique : Université catholique de Louvain (Belgique) : ojs.uclouvain.be

(C) Cet article est mis à disposition selon les termes de la Licence Creative Commons Attribution, Pas d'Utilisation Commerciale 4.0 International. http://creativecommons.org/licenses/by-nc/4.0/

Éditeur : Émulations - Revue de sciences sociales / Presses universitaires de Louvain https://ojs.uclouvain.be/index.php/emulations

ISSN électronique : $1784-5734$

UCL PRESSES

UNIVERSITAIRES 


\title{
Rémi Dormois - Les politiques urbaines. Histoire et enjeux contemporains
}

\begin{abstract}
Antoine Lévêque ${ }^{1}$
Recensé: Rémi Dormois, Les politiques urbaines. Histoire et enjeux contemporains, Rennes, Presses universitaires de Rennes, 2015, 312 p.

Parler de politiques urbaines semble aujourd'hui aller de soi, tant certaines disciplines, comme la science politique, se sont emparées de cet objet. Il n'en a pourtant pas toujours été ainsi. Devant l'évidence d'un champ d'analyse qui s'est imposé compte tenu des évolutions récentes de l'action publique, l'ouvrage de Rémi Dormois propose un tour d'horizon des domaines d'action publique mis en œuvre à l'échelle des villes. En s'autorisant également quelques détours sur l'activité politique qui s'y déploie, il rend compte des différentes acceptions par lesquelles les politiques urbaines ont été appréhendées.

Docteur en science politique et chercheur associé au laboratoire «Environnement, ville, société (EVS - UMR 5600)», R. Dormois a également une formation d'ingénieur des ponts, des eaux et des forêts. Il a travaillé en collectivité territoriale, dans les services de l'État ou encore en bureau d'études et dans le milieu associatif dans l'élaboration et la mise en œuvre de politiques publiques. Ainsi, issu d'un parcours associant une participation directe à l'action publique et un travail de recherche en science politique, l'auteur s'adresse ici à un public large, éventuellement non académique. L'ouvrage se veut donc être un outil pratique et pédagogique remplissant les fonctions d'un manuel. Sa structuration avec des entrées sectorielles (politiques urbaines du logement, de la mobilité, de la culture,...) en est imprégnée et autorise ainsi une entrée thématique. Il s'agit d'abord de restituer quelques grands repères pour quiconque souhaite investir plus avant le champ des politiques urbaines. De ce point de vue, l'apport fondamental de l'ouvrage réside dans l'état de littérature qu'il fournit pour chaque entrée choisie. La succession des chapitres suit également une logique propre, défendue par l'auteur, et permet de voir se dégager une certaine lecture des changements intervenant dans les politiques urbaines. Au-delà des enjeux techniques souvent mis en avant en matière d'urbanisme, d'aménagement ou encore de transport urbain, et au-delà des objectifs régulièrement réaffirmés à propos de la construction de villes durables, $R$. Dormois cherche d'abord à mettre en relief la dimension politique des politiques urbaines. Ainsi, l'auteur s'interroge sur les rapports de forces, sur les coalitions d'acteurs
\end{abstract}

${ }^{1}$ Doctorant en science politique au Laboratoire Triangle (UMR CNRS 5206), Université Lyon 2. 
qui sont parvenus, à travers des contextes singuliers, à imposer un traitement particulier d'un problème considéré alors comme spécifiquement urbain.

Le premier chapitre est consacré aux politiques urbaines de développement économique. Cette entrée en matière permet à l'auteur de revenir sur une dimension structurante qui lui semble peser sur l'ensemble des politiques urbaines, à savoir les mutations de l'organisation du système économique, ainsi que ses répercussions sociales et politiques sur les villes. Ces dernières seraient devenues, dans la période récente, des acteurs-clés de l'économie mondialisée en accompagnant les recompositions contemporaines du capitalisme. Le développement d'une intervention économique par les villes, que l'auteur situe à partir des années 1970, se standardiserait aujourd'hui au niveau des objectifs poursuivis, tournés vers l'attractivité des territoires. Cependant, il nous semble que cette focale historique très contemporaine tend à minimiser non seulement linscription sur le long terme des relations entre élites politiques et économiques locales (Jouve, Lefevre, 1999), mais aussi des velléités plus anciennes de rayonnement de certaines villes (Payre et al., 2013). Ce propos est d'ailleurs nuancé dans les chapitres suivants.

Le deuxième chapitre traite des politiques urbaines sociales. Il débute par un paysage des disparités sociales urbaines présentées comme découlant des tendances abordées dans le premier chapitre. Ensuite, en revenant à l'analyse des politiques publiques, il permet d'opérer un premier recul en soulignant le rôle initiateur de communes progressistes dans la construction du Welfare State, avant de montrer comment la ville est devenue un espace où coexistent différents types de politiques sociales. Certaines d'entre elles sont territorialisées et d'autres territorialement organisées, sans qu'on ne puisse pour autant parler de politiques sociales urbaines, tant le rôle d'acteurs nationaux reste central devant l'absence de cohésion entre les différentes collectivités concernées. Les "grandes étapes» proposées nous semblent, ici, néanmoins un peu abruptes et présentent une vision fonctionnaliste des politiques publiques, pourtant remise en cause en matière de politique de la ville. Aussi, la mise à l'agenda d'une requalification physique lourde des quartiers et d'une lutte contre la délinquance est ici associée à la montée du Front national au début des années 2000, quand d'autres chercheurs, à l'instar de Sylvie Tissot (2007), y voient la continuité d'une réforme profonde des politiques sociales à travers la construction d'un problème spécifique des «quartiers".

C'est également de territorialisation dont il est question dans le troisième chapitre consacré aux politiques de logement. Là encore, si ces dernières ont été un véritable symbole de l'intervention de l'État pendant les Trente Glorieuses, la légitimité de celuici à prendre en charge ce secteur est le fruit d'un processus long, allant de l'émergence de débats nationaux au XIX ${ }^{e}$ siècle sur les conditions de logements des ouvriers dans les villes à l'entreprise des grands ensembles dans le contexte d'après-guerre, en passant par le rôle de maires réformateurs. L’idée d'un basculement «sous la pression de 
l'opinion publique» (p. 89) pour mettre en place cette politique interventionniste centralisée nous parait réductrice, tant certains travaux insistent sur le lent processus d'expérimentation et de structuration du secteur de la construction pour expliquer les choix retenus (Le Goullon, 2014). La mise en perspective introduite par des éclairages européens permet de revenir sur certaines idées reçues. Elle témoigne d'interventionnismes étatiques plus précoces pour certains pays voisins. Enfin, la sortie de cette période fordiste, devant l'émergence d'un nouveau référentiel au tournant des années 1980, engage un repositionnement de l'État dans un rôle de régulateur et d'incitateur, laissant à l'acteur privé la production de logements et, à l'échelon local, la construction de politiques urbaines de l'habitat.

$\mathrm{Au}$ cœur de l'ouvrage, le quatrième chapitre, portant sur les politiques urbaines d'aménagement, nous apparaît le plus stimulant. Le séquençage historique proposé nous renvoie d'abord à la naissance de l'urbanisme au travers d'initiatives portant la nécessité d'une organisation rationnelle du développement des villes. En s'appuyant sur la promotion de savoirs et de méthodes scientifiques, cette démarche s'inscrit dans une rhétorique du progrès social. Devant l'absence d'application des premiers plans d'urbanisme au début du siècle dernier, les promoteurs de l'urbanisme se détournent des maires, accusés d'entretenir des intérêts particuliers, au profit de l'État et de la mise en place de démarches centralisées. Le consensus qui émerge alors trouve une traduction concrète dans les «ordonnances-lois » de 1943, non soumises au vote du Parlement, qui jettent les bases des politiques d'aménagement fordistes, organisant l'ensemble du territoire national pour reconstruire et moderniser «la nation ». La création du Ministère de l'Équipement en 1966 consacre cette prise en main par l'État et ses ingénieurs des ponts et chaussées, aux dépens des architectes et urbanistes. S’opère alors une dépolitisation de l'urbanisme au moyen notamment d'un remplacement sémantique. La notion d'« aménagement» est préférée à celle d'«urbanisme», et il s'agit de programmer une «production urbaine» technicisée plutôt que de mettre en place une «politique urbaine». L'usage d'indicateurs économiques et de la prospective permet « d'esquiver le traitement de problèmes porteurs de luttes politiques » (p. 127). La remise en cause de cette organisation est adossée à la critique de l'urbanisation fonctionnaliste qu'incarnent très largement les grands ensembles. L'impact de la loi d'orientation foncière et les premiers signes d'une crise économique globale ne permettant pas à l'État d'assurer seul le financement des programmes, tandis que le décalage entre ses prévisions et le recensement de 1975 concourent à l'émergence de nouvelles conceptions reposant davantage sur les acteurs locaux. Le référentiel néolibéral est au cœur de la planification territoriale stratégique qui s'impose alors peu à peu à travers le renouvellement des objectifs et des méthodes. Centrés sur le renforcement de l'attractivité des villes, ces derniers concourent à une concentration des budgets sur « une géographie prioritaire du développement» (p. 137). Des initiatives pilotes, telles que la révision du schéma directeur de l'agglomération lyonnaise, se développent dans les années 1980 en mettant au premier plan des structures intercommunales, avant de se diffuser plus 
largement dans la décennie suivante. L'exemple britannique montre un tournant plus brutal encore, visant à rétablir les mécanismes de marché dans les politiques urbaines via différentes mesures du gouvernement conservateur de Margaret Thatcher. Imposé en Angleterre, le développement des partenariats publics-privés est aussi fortement encouragé en France par des dispositifs faisant de l’aménagement un instrument de « gouvernance urbaine ». Au total, la catégorie «aménagement » apparaît chargée de représentations des enjeux urbains qui se succèdent selon les rapports fluctuant entre acteurs publics (experts, hauts fonctionnaires, maires,...) et privés (consultants, promoteurs immobiliers, dirigeant d'entreprises,...)

Les politiques de mobilité font également l'objet d'un éclairage important. Après avoir dressé un panorama des caractéristiques d'une mobilité urbaine, R. Dormois propose un découpage en trois temps des grandes inflexions ayant rythmé cet objet d'action publique. D'abord réduit à la gestion ainsi qu'au développement des réseaux de transport collectif et routier, le traitement des déplacements se serait peu à peu davantage rapproché de l'urbanisme à la faveur d'une approche plus globale et transversale. Le détour sur l'évolution des moyens d'expertises et des acteurs qui les détiennent, d'abord par les services de l'État sur les secteurs qu'il investit, puis au sein des collectivités et plus encore des structures intercommunales (dont l'acceptabilité s'est souvent appuyée sur l'intégration de communes au réseau de transport urbain), est particulièrement éclairant quant aux mécanismes de formulation des solutions. Celles-ci sont également continuellement investies par des acteurs privés, exploitant les réseaux, dont les intérêts dépendent des choix publics. Il semble néanmoins que l'auteur reprend ici à son compte une tradition de recherche proche de l'évaluation de ces politiques en adoptant un discours normatif. Lorsqu'il conclut que « les politiques de mobilité n'accordent pas assez de place à des dispositifs d'action agissant du côté de la demande» (p. 181), à la suite notamment de Jean-Marc Offner (2006), sa posture est prescriptive alors qu'il revendique par ailleurs le fait de s'en écarter. En l'occurrence, ce discours trouve aujourd'hui une réception dans l'action publique alimentant le développement de "nouveaux services à la mobilité» qui laissent davantage place aux acteurs privés. Il semble paradoxal de prétendre ici pouvoir objectiver la demande de mobilité, tant celle-ci découle de pratiques et de représentations sociales (Dezalay, Gueissaz, Maillet, 2007), tout en plaidant pour le maintien d'« une analyse politiste et sociologique » à même de percevoir la " priorisation sociale et spatiale » (p. 181) derrière les politiques de mobilité (Reigner, 2013).

Enfin, les trois derniers chapitres portent sur des politiques urbaines que R. Dormois qualifie de moins « classiques », à savoir les politiques urbaines d'environnement, de la culture et des relations internationales. À rebours des représentations courantes, l'apport majeur nous semble de nouveau résider dans la restitution de l'épaisseur historique que recouvre l'investissement des villes sur ces thèmes. Aussi, des inflexions majeures marquent l'appréhension de ces thématiques par les municipalités. Portée tour 
à tour par différents acteurs qui entrent en concurrence, la façon d'envisager ces problèmes urbains et d'y rattacher des enjeux a évolué. Par exemple, si les villes investissent dès le XIX ${ }^{\mathrm{e}}$ siècle des réseaux transnationaux, court-circuitant ainsi les États, c'est d'abord pour répondre à des objectifs proprement politiques. En revanche, à partir des années 1980, les actions entreprises à cette échelle semblent trouver leur motivation dans la poursuite d'un développement économique. Les dispositifs saisis et le rapport aux institutions supranationales s'en trouvent renouvelés. À la faveur d'un processus d'institutionnalisation, les politiques urbaines d'environnement se sont, quant à elles, peu à peu éloignées des groupes militants qui les ont fait émerger, opérant du même coup une «sélection parmi les défis environnementaux» (p. 204) dont la justification produite par l'expertise répond aussi d'enjeux économiques. En outre, l'auteur mobilise davantage une sociologie des professions sur les politiques urbaines de la culture en reprenant notamment les travaux de Vincent Dubois (2004). Alors que la politisation de la culture, d'abord limitée à quelques municipalités communistes, se répand plus largement dans les agendas locaux à partir des années 1970, le développement d'actions s'accompagne de la croissance d'une administration propre qui amorce un processus de professionnalisation. Aux objectifs socio-éducatifs portés par des militants succède l'organisation d'évènements pris en charge par des professionnels qui dépolitisent les équipements culturels pour en faire "de simple[s] lieu[x] offrant des services et des biens culturels à des consommateurs» (p. 213). Ces inflexions s'accompagnent ensuite d'une orientation économique qui met la culture au service des politiques urbaines de compétitivité et d'attractivité selon une logique d'encastrement. Liés à l'affirmation plus générale d'une politique urbaine néolibérale, ces bouleversements renvoient également à l'influence de différents niveaux de gouvernement, dont notamment les incitations de l'Union européenne en faveur d'une compétition entre les territoires.

De cet ouvrage on regrettera cependant quelques raccourcis empruntés par l'auteur. Les courtes introductions des chapitres présentent parfois des découpages temporels discutables, qui sont néanmoins nuancés dans le développement de l'argumentaire. Bien des concepts et notions mobilisés ne font pas toujours l'objet de définitions préalables, tels qu'« institutionnalisation », "référentiel », "métropolisation» ou encore "gouvernance». Ce sont des termes polysémiques, dont l'emploi par les acteurs des politiques urbaines n'est pas neutre et produit des effets propres, comme l'auteur l'explique lui-même à propos de l'usage de la notion d'« aménagement ». À cela, ajoutons le non-développement systématique des sigles (ONZUS, DRAC, ANAH,...), malgré la présence d'un glossaire en fin d'ouvrage. Ce sont là des éléments qui rendent moins efficace la portée pédagogique de l'ouvrage. Enfin, le renforcement des définitions, la précision des approches mobilisées et leur inscription disciplinaire, rarement précisée pour mobiliser des auteurs, auraient permis de mieux saisir les enjeux des controverses scientifiques entourant les politiques urbaines. 


\section{Bibliographie}

BARDON A. (2014), Les arguments religieux dans la discussion politique : une théorie de la justification publique, thèse de doctorat, Paris, Institut d'études politiques.

BECKFORD J. (2012), «SSSR Presidential Address: Public Religions and the Postsecular: Critical Reflections », Journal for the Scientific Study of Religion, vol. 51, n 1, pp. 1 19.

HABERMAS J. (2008), « Qu’est-ce qu’une société “post-séculière” ?», Le Débat, vol. 5, n 152 , p. 4-15.

SÉNAC R. (2015), L'égalité sous conditions. Genre, parité, diversité, Paris, Presses de Sciences Po (« Académique»).

Dezalay T., Guessaz A., Maillet C. (2007), «Réalité et perception des dimensions spatiales et socio-culturelles de l'accessibilité des services collectifs", EspacesTemps.net. URL : http://www.espacestemps.net/en/articles/realite-etperception-des-dimensions-spatiales-et-socio-culturelles-de-lrsquoaccessibilitedes-services-collectifs-en

JOUVE B., LEFEVRE C. (1999), «De la gouvernance urbaine au gouvernement des villes ? Permanence ou recomposition des cadres de l'action publique en Europe », Revue française de science politique, vol. 49, $\mathrm{n}^{\circ}$ 6, p. 835-854.

Dubois V. (2004), "Dilemmas of the institutionalisation process. From cultural mobilisation to cultural policies », International Journal of Cultural Policy, vol. 10, $\mathrm{n}^{\circ}$ 3, p. 331-349.

LE GOULLON G. (2014), Les grands ensembles en France. Genèse d'une politique publique (1945-1962), Paris, Comité des travaux historiques et scientifiques (« histoire»).

OfFNER J.-M. (2006), Les plans de déplacements urbains, Paris, La Documentation française (« Le point sur »).

PAYRE R.. (dir.) (2013), Lyon, ville internationale : la métropole lyonnaise à l'assaut de la scène internationale, 1914-2013, Lyon, Libel (« SHS »).

REIGNER H. (2013), Sous les pavés de la qualité urbaine. Gouvernement des territoires, gouvernement des conduites et formes renouvelées de la domination dans la ville néohygiéniste, mémoire d'habilitation à diriger des recherches, Paris, Institut d'études politiques de Paris.

Tissot S. (2007), L'état et les quartiers. Genèse d'une catégorie de l'action publique, Paris, Seuil («Liber»). 
